Southern Illinois University Carbondale

OpenSIUC

Publications

Department of Physics

$3-2006$

\title{
Structural and Mechanical Properties of TaZrN Films: Experimental and ab initio Studies
}

S M. Aouadi

Southern Illinois University Carbondale

Follow this and additional works at: http://opensiuc.lib.siu.edu/phys_pubs

(C) 2006 American Institute of Physics

Published in Journal of Applied Physics, Vol. 99 No. 5 (2006) at 10.1063/1.2178394

Recommended Citation

Aouadi, S M. "Structural and Mechanical Properties of TaZrN Films: Experimental and ab initio Studies." (Mar 2006).

This Article is brought to you for free and open access by the Department of Physics at OpenSIUC. It has been accepted for inclusion in Publications by

an authorized administrator of OpenSIUC. For more information, please contact opensiuc@lib.siu.edu. 


\title{
Structural and mechanical properties of TaZrN films: Experimental and $a b$ initio studies
}

\author{
S. M. Aouadia) \\ Department of Physics, Southern Illinois University, Carbondale, Illinois 62901-4401
}

(Received 9 September 2004; accepted 25 January 2006; published online 3 March 2006)

\begin{abstract}
This paper reports on the growth and characterization of the structural and mechanical properties of tantalum zirconium nitride films and the subsequent simulation of these properties using an ab initio calculation based on density functional theory (DFT) within the generalized gradient approximation. The films were deposited by reactive unbalanced magnetron sputtering and their physical and chemical properties were studied by means of x-ray diffraction (XRD), Rutherford backscattering (RBS), and nanoindentation. XRD revealed that these films formed a solid solution and that the lattice constant decreased linearly with Ta content. RBS provided the elemental composition of the films. Nanoindentation was used to evaluate the hardness and the elastic modulus. The hardness was found to have high values for a $\mathrm{Ta} /(\mathrm{Ta}+\mathrm{Zr})$ of $30 \%$ and $100 \%$. The elastic modulus was found to increase monotonically with Ta content. The intrinsic elastic constants were calculated using DFT and the results were compared to the experimental values. A correlation between the computational and the experimental Young's modulus was established. However, the trends observed for the measured hardness and the calculated shear modulus were not in agreement. This disagreement was due to the prominent extrinsic component of the hardness for these materials. (C) 2006 American Institute of Physics. [DOI: 10.1063/1.2178394]
\end{abstract}

\section{INTRODUCTION}

Binary transition metal nitride $\left(M_{e} \mathrm{~N}\right)$ coatings have been largely used as coatings for wear protective applications in the last three decades. ${ }^{1-3}$ They form a rocksalt structure when the transition metal belongs to the IVb or $\mathrm{Vb}$ group in the periodic table. At the nanoscale level, they display three types of bonding characteristics: metallic, ionic, and covalent. ${ }^{4}$ This unusual combination of bonding mechanisms manifests itself in their macroscopic properties. The addition of a second transition metal element from the same group usually forms a single-phase ternary material (solid solution) for nearly stoichiometric films (the nitrogen concentration is kept around 50\%). Examples of such structures that have been investigated in the literature include $\mathrm{TiZrN}^{5-7}$ NbTiN, ${ }^{7,8}$ TiMgN $^{9}$ and CrAlN. ${ }^{10}$ Ternary nitride solid solutions were found to have hardness, wear resistance, melting properties, and corrosion resistance that depend on the type and concentration of the additional element. Enhancement in the properties of these materials may be achieved when the "right" element and the "right" concentration are selected. Understanding these effects is critical to the selection of coating materials for engineering applications. It is well known that hardness and elastic modulus may be used to assess the mechanical properties of materials, including resistance to plastic deformation. ${ }^{11}$ One approach to understand the improvement in mechanical properties through alloying and to predict the degree of improvement is to carry out theoretical calculations which may provide the essential guidance in identifying the optimization process of coating materials with the desired properties.

${ }^{a)}$ FAX: 618-453-1056; electronic mail: saouadi@physics.siu.edu
Very recently, our group has produced nanocrystalline $\mathrm{TaZrN}$ as a protective coating against wear for biomedical and other tribological functions. ${ }^{11}$ These materials were deposited by reactive unbalanced magnetron sputtering and the impact of elemental composition on the structural, chemical, optical, and mechanical properties was investigated. It was found that these materials formed a single-phase rocksalt structure and that their hardness was greatly enhanced due to the addition of $\mathrm{Ta}$ to $\mathrm{ZrN}$ by substitution. The current study reports on $a b$ initio density functional theory calculations that were performed to investigate the effect of alloying with $\mathrm{Ta}$ on the intrinsic mechanical properties of $\mathrm{ZrN}$. The experimental mechanical properties deduced from nanoindentation measurements and the calculated ones were compared.

\section{EXPERIMENTAL DETAILS}

TaZrN films, with a thickness of $1.5 \mu \mathrm{m}$, were deposited by reactive unbalanced magnetron dc sputtering on polished $\mathrm{Si}$ (111) wafers. The films were produced using a bias voltage of $-70 \mathrm{~V}$, a substrate temperature of $-440{ }^{\circ} \mathrm{C}$, and a flow rate of nitrogen of 1.5 SCCM (SCCM denotes cubic centimeter per minute at STP). The power to the $\mathrm{Zr}$ and $\mathrm{Ta}$ targets was varied as shown in Table I. The crystallographic structure of the films was analyzed using $1.54 \AA$ wavelength $\mathrm{Cu} K \alpha$ radiation with a Rigaku Geigerflex D-MAX x-ray diffractometer and a graphite diffracted beam monochromator. Elemental composition was determined using Rutherford backscattering and the results of the chemical analysis are listed in Table I. The Rutherford backscattering (RBS) probe consisted of $2 \mathrm{MeV} \mathrm{He}{ }^{+}$ions incident at an angle of $22.5^{\circ}$ relative to the sample surface normal with the detector set at a $150^{\circ}$ scattering angle. Film hardness was measured using a computer controlled "MTS Systems Corporation Nanoin- 
TABLE I. Deposition conditions and elemental composition of sputtered TaZrN samples.

\begin{tabular}{ccccccc}
\hline \hline Sample & $\begin{array}{c}\text { Ta target } \\
\text { power }(\mathrm{W})\end{array}$ & $\begin{array}{c}\text { Zr target } \\
\text { power }(\mathrm{W})\end{array}$ & $\mathrm{Ta}(\%)$ & $\mathrm{Zr}(\%)$ & $\mathrm{N}(\%)$ & $\mathrm{Ta} /(\mathrm{Ta}+\mathrm{Zr})(\%)$ \\
\hline S1 & 0 & 70 & 0 & $43 \pm 2$ & $57 \pm 2$ & 0 \\
S2 & 9 & 65 & $7 \pm 1$ & $37 \pm 2$ & $56 \pm 2$ & 16 \\
S3 & 13 & 60 & $10 \pm 1$ & $36 \pm 2$ & $53 \pm 2$ & 22 \\
S4 & 20 & 56 & $14 \pm 1$ & $34 \pm 2$ & $60 \pm 2$ & 29 \\
S5 & 27 & 50 & $18 \pm 1$ & $28 \pm 2$ & $54 \pm 2$ & 39 \\
S6 & 34 & 46 & $24 \pm 1$ & $27 \pm 2$ & $59 \pm 2$ & 47 \\
S7 & 60 & 15 & $35 \pm 1$ & $10 \pm 2$ & $55 \pm 3$ & 77 \\
S8 & 80 & 0 & $45 \pm 2$ & 0 & $55 \pm 2$ & 100 \\
\hline \hline
\end{tabular}

denter XP" nanomechanical testing system using loads of $1500 \mu \mathrm{N}$. A more detailed description of the sputtering system and characterization techniques in addition to the thin film fabrication and testing procedures are available elsewhere. ${ }^{11}$

\section{COMPUTATIONAL METHOD}

The ground state energies, lattice parameter, elastic constants, and electronic structure of $\mathrm{TaZrN}$ were calculated within the density functional theory ${ }^{12}$ (DFT) formalism using Cambridge sequential total energy package (CASTEP) software. ${ }^{13-15}$ CASTEP uses a total energy plane wave pseudopotential method. In the mathematical model of the material, CASTEP replaces ionic potentials with effective potentials acting only on the valence electrons in the system. The electronic wave functions were expanded with a plane wave basis setup to a plane wave cut-off energy sufficient for convergence, which varies depending on the convergence of each pseudopotential. The electronic energies were mapped to a set of special $k$ points in the reduced Brillouin zone and the number of $k$ points was determined by the spacing in the reciprocal space. A small $k$ point spacing yields a higher number of $k$ points and more accurate results, but it is also more computationally intensive. The exchange-correlation functional used in this study was the gradient corrected local density approximation of Perdew and Wang (GGA-PW91). ${ }^{16}$ In order to achieve a high level of convergence of the total energy, a high-energy cut-off was chosen. The plane wave cut-off was $330 \mathrm{eV}$. The total self-consistent field (SCF) energy change was $5 \times 10^{-6} \mathrm{eV} /$ atom and the energy between

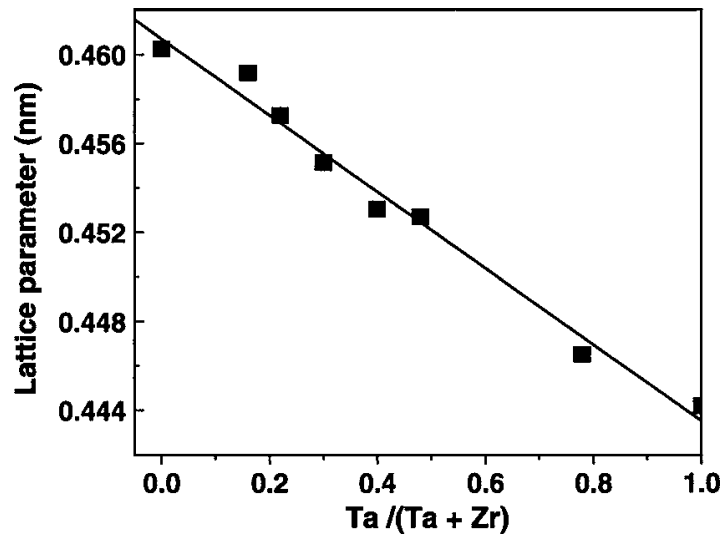

FIG. 1. Experimental lattice parameter as a function of tantalum content. optimization steps was $5 \times 10^{-5} \mathrm{eV} /$ atom. An eight-atom cubic unit cell model with rocksalt structure was used to calculate the lattice parameter, the elastic coefficients, the Mulliken charges, and the electron densities. In order to study the effect of alloying on the various properties of the films, one or more $\mathrm{Zr}$ atoms were replaced by Ta atom(s). The author assumes that the Ta elements are uniformly distributed in the lattice.

\section{RESULTS AND DISCUSSION}

\section{A. Lattice constants}

XRD data for the TaZrN samples ${ }^{11}$ indicated planar spacings typical of the NaCl-type structure of $\mathrm{TaN}$ and $\mathrm{ZrN}^{17-20}$ The data revealed strong (111) and (200) preferred orientations though other diffraction lines were also observed. The lattice structure of $\mathrm{TaZrN}$ consisted of a solid solution whereby $\mathrm{Zr}$ atoms were substituted by Ta atoms. As a result, a general shift in the $2 \theta$ positions towards higher $d$ values was observed with increasing the tantalum content in the lattice. Figure 1 shows the lattice parameter as a function of tantalum content, represented by the quotient $\mathrm{Ta} / \mathrm{Zr}$ $+\mathrm{Ta}$ ), which clearly indicated that the deposited films formed a single phase. By fitting each diffraction peak to a pseudo-Voigt function, ${ }^{21}$ the average grain sizes were calculated from the widths of the XRD peaks using the Scherrer formula and are shown in Fig. 2. This figure indicated that the grain size remained the same (around $24 \mathrm{~nm}$ ), except for samples with a high Ta content, for which the grain size decreased dramatically to a value of $14 \mathrm{~nm}$.

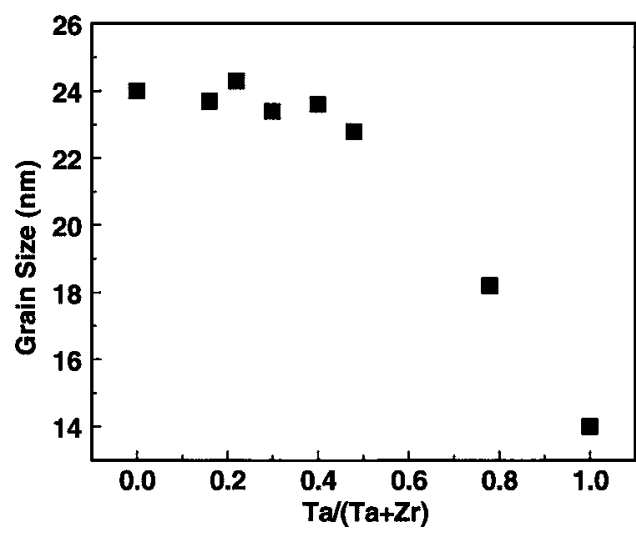

FIG. 2. Grain size as a function of Ta content. 


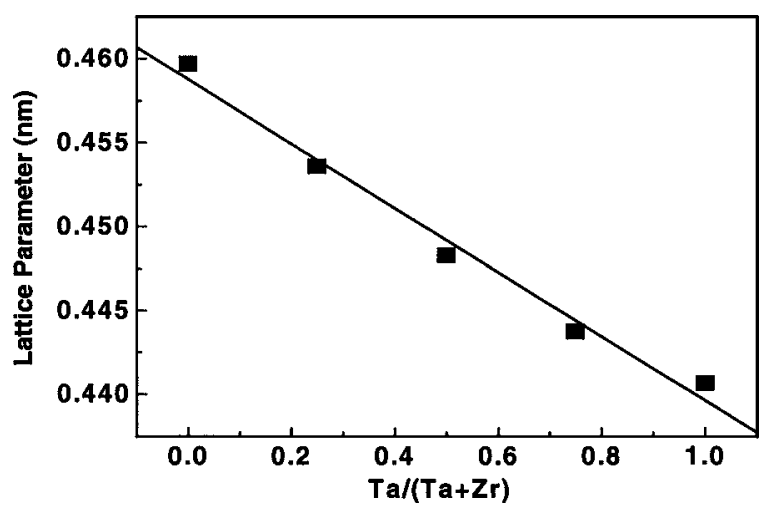

FIG. 3. Theoretical lattice parameter as a function of tantalum content

The calculated total energies $E_{\text {tot }}$ varied as a function of the lattice parameter for $\mathrm{ZrN}$-based nitrides. Equilibrium lattice constants were determined from the minima of the curves and are shown in Fig. 3. This figure revealed that the experimental values for the equilibrium lattice constants were larger by about $0.3 \%$ than the GGA-PW91 calculated ones. Similar observations were reported for other binary nitrides using the same exchange-correlation potential. ${ }^{4}$ In addition, the lattice parameter of thin films is usually higher than that of bulk materials for the following reasons: ${ }^{22}$

- the difference in the thermal expansion coefficient between the film and the substrate,

- the presence of impurities such as oxygen and argon,

- line and point defects introduced by the nonequilibrium growth conditions,

- nonstoichiometric growth conditions, and

- the existence of high internal stresses generated by the presence of a high density of grain boundaries and defects.

\section{B. Elastic constants}

The hardness and elastic modulus of the TaZrN samples were measured using a total of nine indentations. The mean values and standard deviation of hardness and elastic modulus were computed. Figure 4 shows the film hardness and elastic modulus as a function of tantalum content. The hardness of the film increases gradually with the Ta content to a maximum value of $37 \pm 3 \mathrm{GPa}$ when Ta content reaches $29 \%$

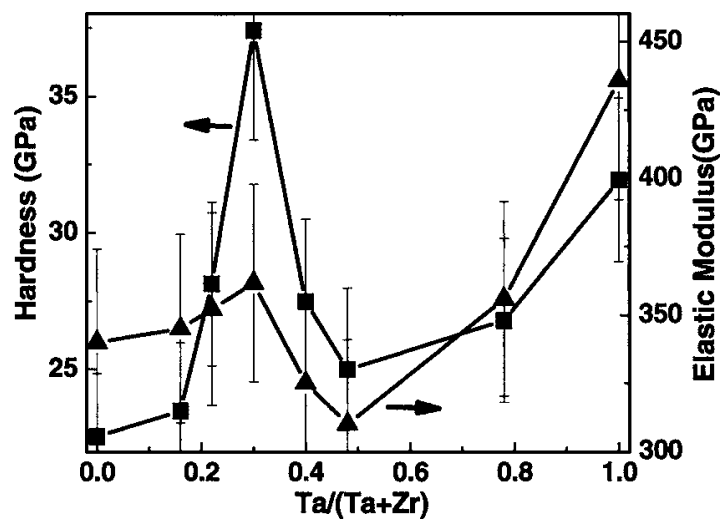

FIG. 4. Hardness and elastic modulus as a function of tantalum content. [in terms of $\mathrm{Ta} /(\mathrm{Ta}+\mathrm{Zr})$ content]. This value is approximately 1.7 times higher than that of pure $\mathrm{ZrN}$ deposited under the same conditions. Further alloying with Ta resulted in a decrease in hardness values. The hardness values increased subsequently as the composition approached that of pure TaN (Ta became the predominant transition metal). The elastic modulus did not vary significantly for low Ta content and increased when the Ta content exceeded $50 \%$.

Hardness $(H)$ is commonly defined as the resistance of a material to deformations. The hardness $H$ of a material may be written as follows: ${ }^{23}$

$$
H=H_{\text {int }}+H_{\text {ext }},
$$

where $H_{\text {int }}$ and $H_{\text {ext }}$ are the intrinsic and the extrinsic contributions to the hardness, respectively. $H_{\text {int }}$ is due to the nature of the bonding structure of a material, while $H_{\text {ext }}$ is a result of the "defects" in the material, which include solid solution, precipitation, grain boundary, work hardening, and so on, and hence, strongly depends on the microstructure of the films. A material's Young's modulus $E$ is a measure of the second derivative of the bond energy at the equilibrium interatomic distance $x_{0}$. $E$ becomes larger for large binding energies and short bond distances $x_{0}$. Other quantities that define the mechanical properties of a material include the bulk modulus $B$, which measures the resistance of a material to a volume change and provides an estimate of its elastic response to a hydrostatic pressure, and the shear modulus $G$, which describes the resistance of a material to a shape change and plays an important role in the elastic theory of dislocations. Hence, the hardness of a material should be closely correlated to how readily a large number of dislocations are generated and are able to move throughout a solid in response to a shear stress. The shear modulus is correlated to the hardness of a material, which was confirmed experimentally as being the best hardness predictor. ${ }^{23-25}$ Values of elastic constants provide valuable information about the bonding characteristic between adjacent atomic planes and the anisotropic character of the bonding and structural stability. $\mathrm{Pugh}^{26}$ introduced the ratio between the bulk and shear modulus, $G / B$, for polycrystalline phases, as a measure of fracture/toughness in metals. A high (low) $G / B$ value is associated with brittleness (ductility). A similar relationship that has recently been used to predict the wear resistance performance of protective coatings is the ratios $H / E$ and/or $H^{3} / E^{2}{ }^{27}$ In this case, a high value of $H / E$ or $H^{3} / E^{2}$ is associated with a high resistance to plastic deformation.

The theoretical behavior of a completely asymmetric material is specified by 21 independent elastic constants, while for an isotropic material, the number is 2. For a rocksalt structure (cubic symmetry), there are three independent elastic constants, namely, $C_{11}, C_{44}$, and $C_{12}$. The cubic symmetry was maintained for all substitutions of the $\mathrm{Zr}$ atoms with Ta atoms except when the number of substitution was 2 $(\mathrm{Ta} / \mathrm{Ta}+\mathrm{Zr}=0.5)$. In this case, the lattice was tetragonal. The bulk modulus $B$ may be directly derived from the second derivative of the total energy $E_{\text {tot }}$ as a function of an isotropic volume change. $B$ can also be expressed as a linear combination of two elastic constants as follows: 


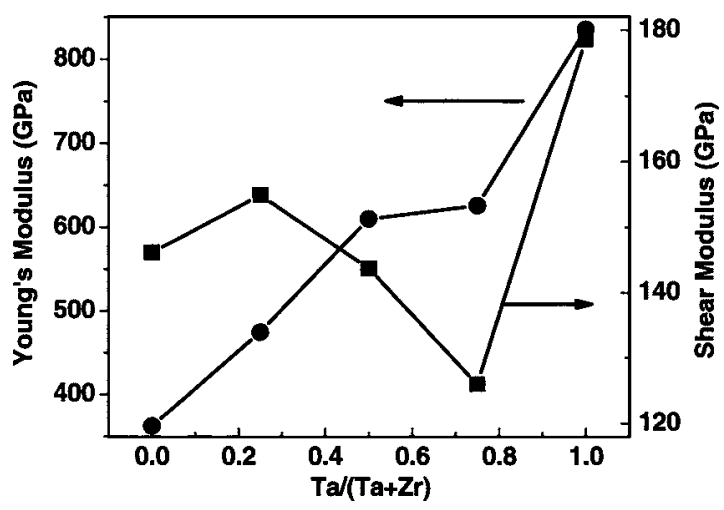

FIG. 5. Calculated Young's modulus and shear modulus.

$$
B=\frac{C_{11}+2 C_{12}}{3} .
$$

The determination of the stress strain distribution in a polycrystalline aggregate with respect to an external load can be established by equating the uniform strain in the polycrystal to the external strain in the Voigt approximation. ${ }^{23,28}$ Hence, the shear modulus $G$, Young's modulus $E$, and Poisson's ratio $\nu$ can be calculated from the following relations:

$$
\begin{aligned}
G & =\frac{3 C_{44}+C_{11}-C_{12}}{5}, \\
E & =\frac{9 B G}{3 B+G}, \\
\nu & =\frac{1}{2}\left(1-\frac{E}{3 B}\right) .
\end{aligned}
$$

For the lattice with tetragonal symmetry, the shear modulus was calculated using the following expression:

$$
G=\frac{1}{15}\left(2 C_{11}+C_{33}-C_{12}-2 C_{13}+6 C_{44}+3 C_{66}\right) .
$$

The elastic constants were computed using CASTEP and the $G$ and $E$ values were calculated from the above equations for TaZrN with various Ta contents and are shown in Fig. 5. The general trends for the computed $G$ and measured $H$ values shown in Figs. 4 and 5 are in disagreement except for the extreme cases that correspond to the binary $\mathrm{ZrN}$ and $\mathrm{TaN}$ systems. The theoretical $G$ values did not display a substantial increase in hardness for $\mathrm{Ta} /(\mathrm{Ta}+\mathrm{Zr})$ in the vicinity of $30 \%$. The theoretical shear modulus for a film with $30 \%$ $\mathrm{Ta} /(\mathrm{Ta}+\mathrm{Zr})$ content may be approximated by a weighted average of the values for contents of $25 \%$ and $50 \%$ and is, therefore, not expected to have a substantial value. ${ }^{29}$ The increased hardness values at these compositions are therefore due to the extrinsic contribution to hardness. Extrinsic hardness mechanisms in nanocrystalline $\mathrm{TaZrN}$ solid solutions include (1) grain boundary hardening, (2) solid solution hardening, (3) limited segregation of solutes at grain boundaries, and (4) vacancy hardening. The incorporation of Ta atoms into the $\mathrm{ZrN}$ lattice did not lead to a noticeable decrease in grain size (at least for these compositions), which disqualifies grain boundary hardening as being the reason for the difference between the theoretical and experimental $H$ and $G$ curves. Vacancy hardening falls in the same category, i.e., is disqualified, since all films are overstoichiometric with a nitrogen content that did not vary in a consistent fashion. Obviously, the fact that our films are overstoichiometric will influence their overall mechanical properties. Even if the nominal composition is close to an ideal stoichiometry, a considerable amount of vacancies are present. ${ }^{30}$ One potential candidate that could explain the sudden increase in hardness for a Ta content of $30 \%$ is solid solution hardening since the replacement of $\mathrm{Zr}$ by $\mathrm{Ta}$ atoms can cause lattice distortion and the resulting stress field can react with the elastic stress field of a dislocation. The other potential contributor to the observed hardening is a limited segregation of solutes at grain boundaries, which could be created by the energetic species that bombard the film during the sputtering process.

The theoretical and experimental elastic moduli were more in agreement and showed a similar trend, as shown in Figs. 4 and 5. For single-phase materials, the elastic modulus has been well known to depend on the nature of atomic bonding, but is independent of the microstructure of the materials, because the bonding nature originates from the atomic configuration of each component of the material. At the nanoscale, however, size effects often become more prominent due to very high surface-to-volume ratios. The effective elastic properties are governed not only by classical bulk elastic strain energy but also by the interfaces (or grain boundaries). The main difference between the computational and experimental data was that the theoretical elastic modulus values increased more significantly with Ta content. A difference between the absolute values for $E$ was expected since the deposited films were nanocrystalline and should exhibit a lower elastic modulus than the materials simulated using the $a b$ initio method, which portray a crystalline material. The discrepancy between the trends in the theoretical and experimental data could be due to the nanocrystalline nature of the films and to the presence of defects (mainly vacancies).

\section{Electronic structure}

Figure 6 shows the partial $p$ - and $d$-state densities for (a) $\mathrm{ZrN}$ and (b) TaN. In this figure, the metal $d$ orbitals of $e_{g}$ symmetry hybridize strongly with the $p$ states of nitrogen. The double-peaked structure found between -8 and $-3 \mathrm{eV}$ for $\mathrm{ZrN}$ and between -10 and $-5 \mathrm{eV}$ for $\mathrm{TaN}$ originates from the nonmetal $2 p$ orbitals hybridizing with the metal $d$ orbitals. This hybridization is strongest for the TaN system. The intrinsic hardness and elastic modulus are therefore expected to be higher for TaN than for $\mathrm{ZrN}$, as was demonstrated in Sec. IV B.

\section{Population analysis}

Population analysis in CASTEP is performed using a projection of the plane wave states onto a localized basis using a technique described by Sanchez-Portal et al. ${ }^{31}$ The population analysis of the resulting projected states is then performed using the Mulliken formalism. ${ }^{32}$ This technique is widely used in the analysis of electronic structure calcula- 

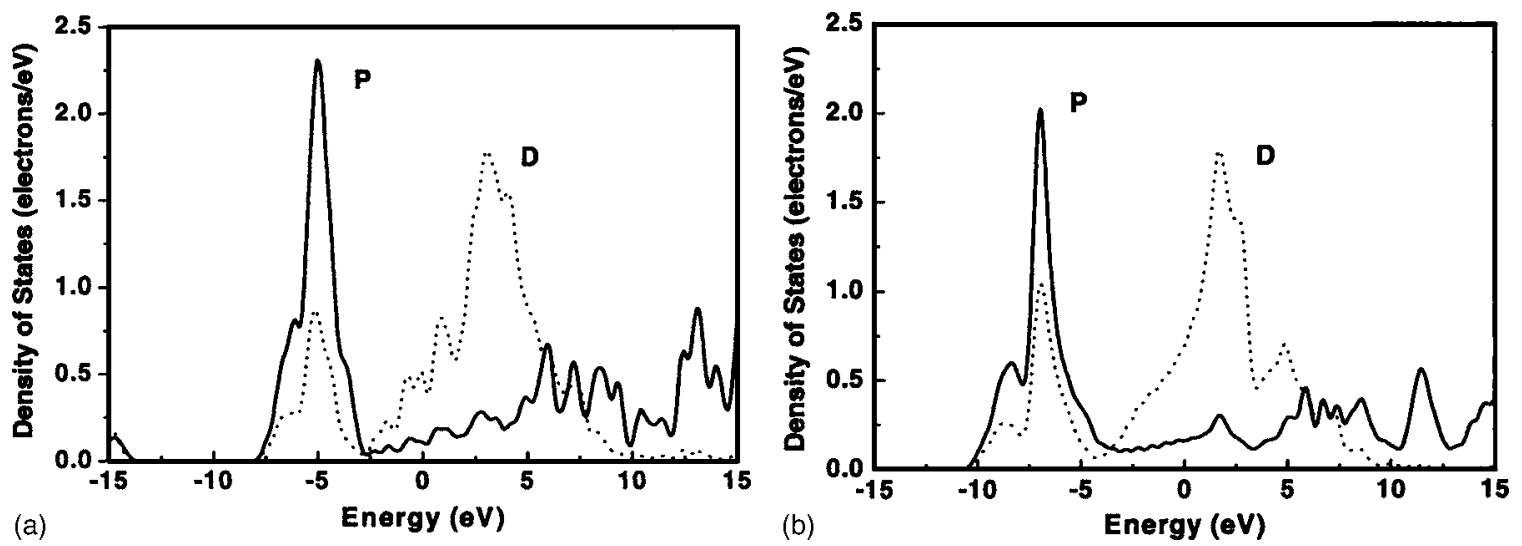

FIG. 6. Partial density of states for (a) $\mathrm{ZrN}$ and (b) TaN.

tions performed with linear combination of atomic orbitals (LCAO) basis sets. In addition to providing an objective criterion for bonding between atoms, the overlap population may be used to assess the covalent or ionic nature of a bond. A high value of the bond population indicates a covalent bond, while a low value indicates an ionic interaction. A further measure of ionicity may be obtained from the effective ionic valence, which is defined as the difference between the formal ionic charge and the Mulliken charge on the anion species. A value of zero indicates a perfectly ionic bond, while values greater than zero indicate increasing levels of covalency. ${ }^{33}$ Table II gives the values of the average bond population and the effective ionic valence charge for the various $\mathrm{TaZrN}$ compositions. This technique offers information on the nature of the bonds formed in the system. The results shown in Table II indicate that the bonds became more covalent with the addition of Ta to the lattice and explain why $\mathrm{TaN}$ is harder than $\mathrm{ZrN}$.

\section{CONCLUSIONS}

Thin films of TaZrN were deposited on Si (111) substrates using unbalanced magnetron sputtering. The films formed a solid solution and the lattice constant was found to increase with the increase in Ta content. The hardness of the films was evaluated using nanoindentation and was found to have a maximum value when the Ta to metal content was about $30 \%$. Theoretical calculations on the structural, mechanical, and electronic properties of these materials are presented in this paper. The calculated lattice constants are in agreement with the experimental data when the generalized gradient approximation (GGA) is used for the exchange and correlation potential. The hardness and shear modulus were

TABLE II. Mulliken charge and population for TaZrN films.

\begin{tabular}{ccc}
\hline \hline $\begin{array}{c}\mathrm{Ta} /(\mathrm{Ta}+\mathrm{Zr}) \\
(\%)\end{array}$ & $\begin{array}{c}\text { Mulliken } \\
\text { charge }\end{array}$ & Population \\
\hline 0 & 0.82 & 0.62 \\
25 & 0.80 & 0.63 \\
50 & 0.79 & 0.65 \\
75 & 0.78 & 0.68 \\
100 & 0.76 & 0.71 \\
\hline
\end{tabular}

in disagreement, especially when the Ta content was in the vicinity of $30 \%$. The substantial increase in the experimental hardness observed for these compositions may be explained by the mechanisms of solid solution hardening and/or phase segregation at grain boundaries. More agreement was observed for the elastic modulus. The experimental and theoretical calculations for hardness and elastic constants concurred for the extreme cases of $\mathrm{TaN}$ and $\mathrm{ZrN}$. TaN is harder due to the stronger hybridization of the nonmetal $2 p$ orbitals with the metal $d$ orbitals (more covalent bond).

\section{ACKNOWLEDGMENTS}

The authors would like to thank Professor I. Petrov, Professor M. Sardela, and Professor M. Williams for XRD and RBS measurements carried out in the Center for Microanalysis of Materials, University of Illinois, which is partially supported by the U.S. Department of Energy under Grant No. DEFG02-91-ER45439. Acknowledgment is extended to Dr. Qingfeng Ge of Southern Illinois University for useful discussions on density functional theory. This research was supported by an award from Research Corporation and by the Materials Technology Center and the Center for Advanced Friction Studies of Southern Illinois University.

${ }^{1}$ W. D. Sproul, Thin Solid Films 107, 141 (1986).

${ }^{2}$ R. A. Andrievski, I. A. Anisimova, V. P. Anisimov, V. P. Makarov, and V. P. Popova, Thin Solid Films 261, 83 (1995).

${ }^{3}$ M. Nose, T. Nagae, M. Yokota, S. Saji, M. Zhou, and M. Nakada, Surf. Coat. Technol. 116, 296 (1999).

${ }^{4}$ T. Amriou, A. Bouhafs, H. Aourag, B. Khelifa, S. Bresson, and C. Mathieu, Physica B 325, 46 (2003).

${ }^{5}$ S. M. Aouadi, J. Chladek, F. Namavar, and S. L. Rohde, J. Vac. Sci. Technol. A 20, 1967 (2002).

${ }^{6}$ M. B. Takeyama, T. Itoi, E. Aoyagi, and A. Noya, Appl. Surf. Sci. 216, 181 (2003).

${ }^{7}$ J. Probst, U. Gbureck, and R. Thull, Surf. Coat. Technol. 148, 226 (2001).

${ }^{8}$ N. N. Iosad, B. D. Jackson, F. Fero, J. R. Gao, S. N. Polyakov, P. N. Dimitriev, and T. M. Klapwijk, Supercond. Sci. Technol. 12, 736 (1999).

${ }^{9}$ O. Banakh, M. Balzer, M. Fenker, and A. Blatter, Thin Solid Films 455456, 650 (2004)

${ }^{10}$ O. Banakh, P. E. Schmid, R. Sanjinés, and F. Levy, Surf. Coat. Technol. 163-164, 57 (2003).

${ }^{11}$ S. M. Aouadi, P. Filip, and M. Debessai, Surf. Coat. Technol. 187, 177 (2004).

${ }^{12}$ R. M. Dreizler and E. K. U. Gross, Density Functional Theory (Springer, Berlin, 1990)

${ }^{13}$ V. Milman, V. Winkler, J. A. White, C. J. Pickard, M. C. Payne, E. V. Akhmatskaya, and R. H. Nobes, Int. J. Quantum Chem. 77, 895 (2000). 
${ }^{14}$ M. C. Payne, M. P. Teter, D. C. Allan, T. A. Arias, and J. D. Joannopoulos, Rev. Mod. Phys. 64, 1045 (1992).

${ }^{15}$ M. D. Segall, P. J. D. Lindan, M. J. Probert, C. J. Pickard, P. J. Hasnip, S. J. Clark, and M. C. Payne, J. Phys.: Condens. Matter 14, 2717 (2002).

${ }^{16}$ Y. Wang and J. P. Perdew, Phys. Rev. B 43, 8911 (1991).

${ }^{17}$ Y. M. Lu, R. J. Weng, W. S. Hwang, and Y. S. Yang, Mater. Chem. Phys. 72, 278 (2001)

${ }^{18}$ D. Pilloud, A. S. Dehlinger, J. F. Pierson, A. Roman, and L. Pichon, Surf Coat. Technol. 174-175, 338 (2003).

${ }^{19}$ H. M. Benia, M. Guemmaz, G. Schemerber, A. Mosser, and J.-C. Parlebas, Appl. Surf. Sci. 200, 231 (2002).

${ }^{20}$ M. S. El-Eskandarany and A. H. Ashour, J. Alloys Compd. 313, 224 (2000).

${ }^{21}$ M. F. Cerqueira, J. A. Ferreira, and G. J. Adriaensses, Thin Solid Films 370, 128 (2000)

${ }^{22}$ J. M. Schneider, A. Voevodin, C. Rebholz, A. Matthews, J. H. C. Hogg, D. B. Lewis, and M. Ives, Surf. Coat. Technol. 74-75, 312 (1995).
${ }^{23}$ M. Mattessini and S. F. Matar, Phys. Rev. B 65, 075110 (2002).

${ }^{24}$ D. M. Teter, MRS Bull. 23, 22 (1998).

${ }^{25}$ M. Hebbache, Solid State Commun. 113, 417 (2000).

${ }^{26}$ S. F. Pugh, Philos. Mag. 45, 823 (1954).

${ }^{27}$ A. Leyland and A. Matthews, Wear 246, 1 (2000).

${ }^{28}$ P. Ravindran, L. Fast, P. A. Korzhavyi, B. Johansson, J. Wills, and O. Eriksson, J. Appl. Phys. 84, 4891 (1998)

${ }^{29}$ S.-H. Jhi, J. Ihm, S. G. Louie, and M. L. Cohen, Nature (London) 399, 132 (1999).

${ }^{30}$ W. Wolf, R. Podloucky, T. Antretter, and F. D. Fisher, Philos. Mag. B 79, 839 (1999).

${ }^{31}$ D. Sanchez-Portal, E. Artacho, and J. M. Soler, Solid State Commun. 95, 685 (1995)

${ }^{32}$ R. S. Mulliken, J. Chem. Phys. 23, 1833 (1955).

${ }^{33}$ M. D. Segall, R. Shah, C. J. Pickard, and M. C. Payne, Phys. Rev. B 54, 16317 (1996). 